\title{
AN AXIAL-SYMMETRIC TRANSONIC FLOW PATTERN ${ }^{1}$
}

\author{
$\mathrm{Br}$ \\ G. GUDERLEY AND H. YOSHIHARA \\ Air Materiel Command, Wright-Patterson Air Force Base
}

1. Introduction. Recently some examples of two-dimensional flow patterns with a free stream Mach number one were given. (Refs. 1 and 2) The basis for their determination was an investigation of the flow at infinity in the physical plane. It is practical to carry out this investigation in the hodograph plane, but in principle the same question can also be dealt with in the physical plane. In this form the discussion can be extended to the case of axial-symmetry. This investigation is carried out in the present paper.

2. The flow pattern at Mach number one. Before we proceed to the mathematical discussion let us first describe qualitatively the flow pattern whose behavior at infinity we are trying to find. Assume we have an axial-symmetric body in a wind tunnel with a circular cross-section. If the tunnel is operating under choking conditions, then upstream of the body the flow is subsonic while downstream it is supersonic. These two regions are separated by a sonic line which proceeds from the surface of the body to the wind tunnel wall. From the supersonic portion of the body two classes of Mach waves will arise; those of the first class start from the body and end at the sonic line, while the waves of the second also start from the body but end at the tunnel wall. There is one Mach wave which separates these two classes, namely, that one which reaches the sonic line at its intersection with the wall. We shall label this wave the limiting Mach wave. It is clear that the subsonic portion of the flow is not completely determined by specifying only the subsonic part of the body. The portion of the supersonic contour along which the Mach waves of the first class originate is also necessary since perturbations propagating along these waves will reach the sonic line and thereby influence the subsonic region. Therefore, the limiting Mach wave forms the boundary of that portion of the supersonic region which is necessary to determine the subsonic field. The flow further downstream represents a purely supersonic problem which may be computed independently by the method of characteristics.

If now the diameter of the test section is increased while the body size is kept constant, then the free stream Mach number upstream of the body will tend to one. Therefore, it is plausible that a limiting flow pattern for an infinite diameter of the tunnel should exist representing the steady flow with the free stream Mach number one. Here also a limiting Mach wave will be present, so that for the determination of the problem one need consider only the flow upstream of the limiting Mach wave.

3. Basic equations. Let $x$ and $y$ be the Cartesian coordinates in a representative meridian plane of an axially symmetric flow, the $x$-axis being in the free stream direction, $a^{*}$ the sonic velocity, $\phi$ the velocity potential, and $\kappa$ the ratio of the specific heats. If one defines a perturbation potential $\varphi$ which gives the deviation from a sonic flow by

$$
\varphi(x, y)=\phi(x, y)-a^{*} x,
$$

then with the approximations usually made in the derivation of the transonic similarity law one obtains the non-linear equation

${ }^{1}$ Received July 15, 1949. This is a condensed version of USAF Tech. Report No. 5783. 


$$
-(\kappa+1) \varphi_{x} \varphi_{x x}+\varphi_{y y}=-y^{-1} \varphi_{y} .
$$

The behavior at infinity of a flow with the free stream Mach number one will be represented by a particular solution of the form

$$
\varphi(x, y)=y^{3 n-2} f(\zeta)
$$

where

$$
\zeta=(\kappa+1)^{-1 / 3} x y^{-n}
$$

and $n$ is a parameter to be determined. The desired solution may be called the basic singularity. Inserting this hypothesis into Eq. (2) one finds that $f$ must fulfill the differential equation,

$$
\left(f^{\prime}-n^{2} \zeta^{2}\right) f^{\prime \prime}+(5 n-4) n \zeta f^{\prime}-(3 n-2)^{2} f=0
$$

Here the primes denote differentiation with respect to $\zeta$.

The discussion of the integral curves of this non-linear equation is facilitated by a group property which it possesses. If a scale transformation is introduced to both $f$ and $\zeta$ in Eq. (5), it is found that the differential equation is invariant with a suitable choice of the scale factors. For a differential equation with this property it is possible to reduce the order of the equation. Indeed if two new variables $s$ and $t$ are introduced by

$$
s=\zeta^{-3} f, t=\zeta^{-2} f^{\prime},
$$

then one will obtain the first order equation

$$
\frac{d t}{d s}=\frac{2 t^{2}+(3 n-4) n t-(3 n-2)^{2} s}{\left(n^{2}-t\right)(t-3 s)} .
$$

If one now solves Eq. (7), then one can obtain the corresponding integral of the second order equation (5) by means of Eqs. (6). In this connection the value of $\zeta$ is found from the integral of Eq. (7) by the following auxiliary equation derived from Eqs. (6), namely,

$$
\frac{d \log \zeta}{d t}=\frac{1}{t-3 s} .
$$

Actually the $s, t$ representation will be used solely for the discussion of the integral curves while the final computation is carried out by means of a numerical integration of the differential equation for $f$.

4. Structure of the integral curves in the $s, t$-system. A survey of the integrals of Eq. (7) is given in Fig. 1. For this purpose the entire $s, t$-plane has been projected onto a hemisphere as shown in Fig. 2 and the hemisphere is then distorted to form the plane circular region of Fig. 1. Infinity in the $s, t$-plane is then represented by the outer circle. The arrows on the integral curves show the direction in which the absolute value of $\zeta$ increases.

As is seen from Eqs. (3) and (6) the sonic line $\left(\varphi_{x}=0\right)$ is represented by the line $t=0$, that is, line $\mathrm{B}^{\prime} \mathrm{AB}$ in Fig. 1 . The subsonic region lies below this line while the supersonic region lies above it.

Along the line $t=n^{2}$ one finds an extremum of $\zeta$. This may be seen by inserting 
the differential equation (7) into Eq. (8) and then setting $t=n^{2}$. Let us examine the significance of an extremal value of $\zeta$. If one travels along an integral curve of the $s, t$-system, then the corresponding lines $\zeta=$ constant sweep a certain region of the physical plane. The occurence of an extremum of $\zeta$ along the integral curve will indicate that one sweeps out a certain region of the flow pattern up to the extremal value of $\zeta$ and then resweeps the same portion of the physical plane. One thus obtains a multivalued physical plane, the line for which $\zeta$ is an extremum being a limiting line. Conse-

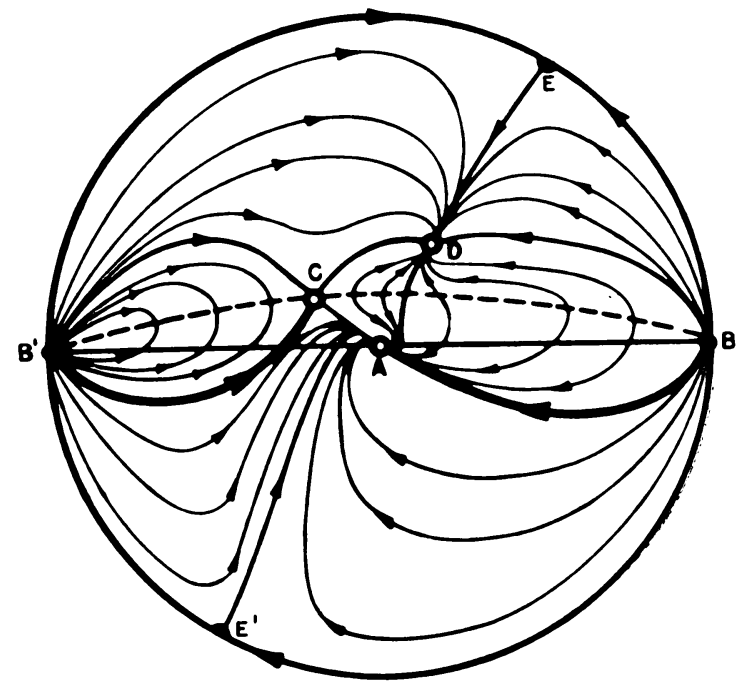

FIG. 1. The integral curves of the $s, t$-system projected upon a hemisphere for $n=4 / 7$

quently all integral curves which cross the line $t=n^{2}$ (dashed line in Fig. 1) are of no physical interest. As will be seen later, the integral curve which goes through point $C$ will be an exception.

Let us consider the singularities of the differential equation (7). The coordinates of the singular points in the finite portion of the $s, t$-plane are

$$
\begin{array}{lll}
s=0, & s=n^{3}(5 n-4)(3 n-2)^{-2}, & s=2 / 9, \\
t=0, & t=n^{2}, & t=2 / 3 .
\end{array}
$$

They are shown in Fig. 1 at points $A, C$, and $D$, respectively.

Point $A$ is a nodal point for all values of $n$ while points $C$ and $D$ may be either a nodal point or a saddle point depending upon the value of $n$. For our problem $n$ will be confined to the range between zero and one because of the requirement of zero perturbation velocities at infinity. For $0<n<2 / 3$ point $C$ will be a saddle point and point $D$ will be a nodal point while for $2 / 3<n<1$ the two points interchange their structures. For $n=2 / 3$ the two points coincide to form a singular point which has both the character of a nodal point and a saddle point.

The singular points at infinity are investigated best by means of the introduction of homogeneous coordinates, $x_{0}, x_{1}$, and $x_{2}$ of Fig. 2. One finds two singular points at infinity. Point $B$ (which is identical with $B^{\prime}$ ) is a combination of a saddle point and a 
nodal point; it is reached as $t$ tends to infinity at a constant value of $s$. Point $E$ is a saddle point, and it is reached by traveling outward along a line $s / t=1$.

The significance of the different singular points is seen if one integrates the differential equation approximately for the neighborhood of the singularities and then determines the behavior of $\zeta$ and the velocity components of the perturbation potential.

It is found that $\zeta$ tends to infinity as one travels towards point $A$ along any of the integral curves. Therefore point $A$ corresponds to the $x$-axis. Among the integral curves which enter point $A$ only one can be expressed in terms of a power series. All other integral curves will contain a logarithmic term. It is found that only for this one integral curve will the velocity component in the $y$-direction tend to zero as one approaches point A.

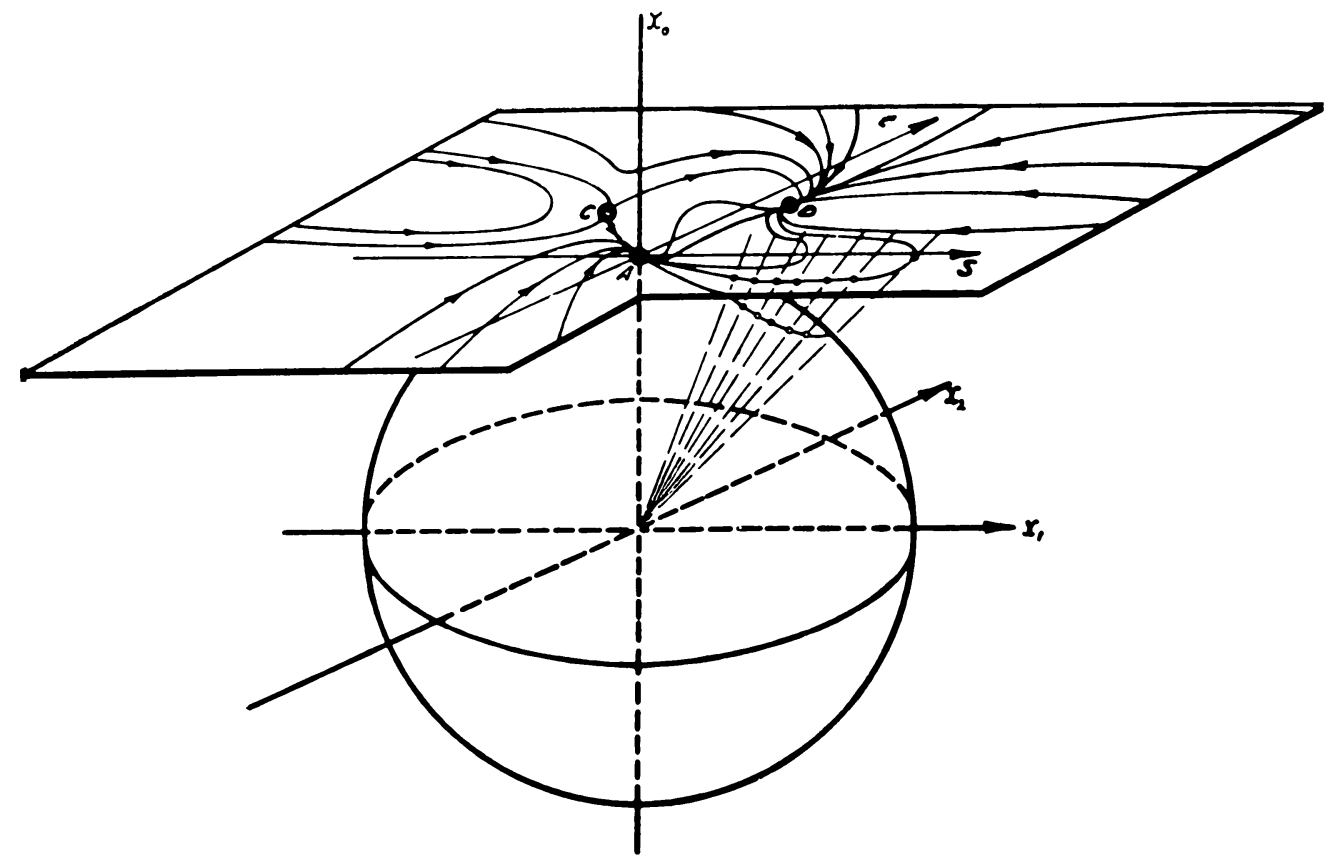

FIG. 2. Orientation of the homogeneous coordinate system.

For all integral curves which enter point $B \zeta$ tends to zero; that is, point $B$ corresponds to the $y$-axis. The velocity components at this point will in general assume finite values with no special restrictions imposed upon them. Since a subsonic velocity cannot jump suddenly to a supersonic velocity or vice-versa, the continuation of a curve which enters point $B$ with a subsonic velocity, for example, must leave point $B^{\prime}$ again with subsonic velocities.

At point $C \zeta$ assumes a finite value. If one rewrites the expressions for the coordinates $s$ and $t$ at this point in terms of the components of the perturbation velocity, then one finds that point $C$ corresponds to a line $\zeta=$ constant which coincides with a Mach wave. Furthermore, it can be shown that all Mach waves which lie upstream of that represented by point $C$ will reach the sonic line in a finite distance from the axis. Therefore, point $C$ will represent the limiting Mach wave described in Section 2.

The other singular points are without interest in the present case. 
5. The determination of the paramenter $n$. It is now possible to describe qualitatively the properties of the integral curve which will represent the desired solution. The integral curve will start at point $A$ which represents the horizontal axis upstream of the body. Only that solution which is regular here can be used since along the negative $x$-axis the $y$-component of the velocity is obviously zero. One will then proceed in the direction of subsonic velocities to point $B$ which corresponds to the $y$-axis. The integral curve now reappears from point $B^{\prime}$ with a subsonic velocity and then crosses the sonic line $B^{\prime} A$ and enters the supersonic region. It should end at point $C$ which represents a line $\zeta=$ constant corresponding to the limiting Mach wave. Naturally for this purpose it is necessary that the value of $n$ be chosen correctly.

It is interesting to see what happens to the solution in the case that the value of $n$ has been chosen incorrectly. The course of the integral curve from point $A$ to point $B$. will be qualitatively the same for values of $n$ in the neighborhood of the desired one.

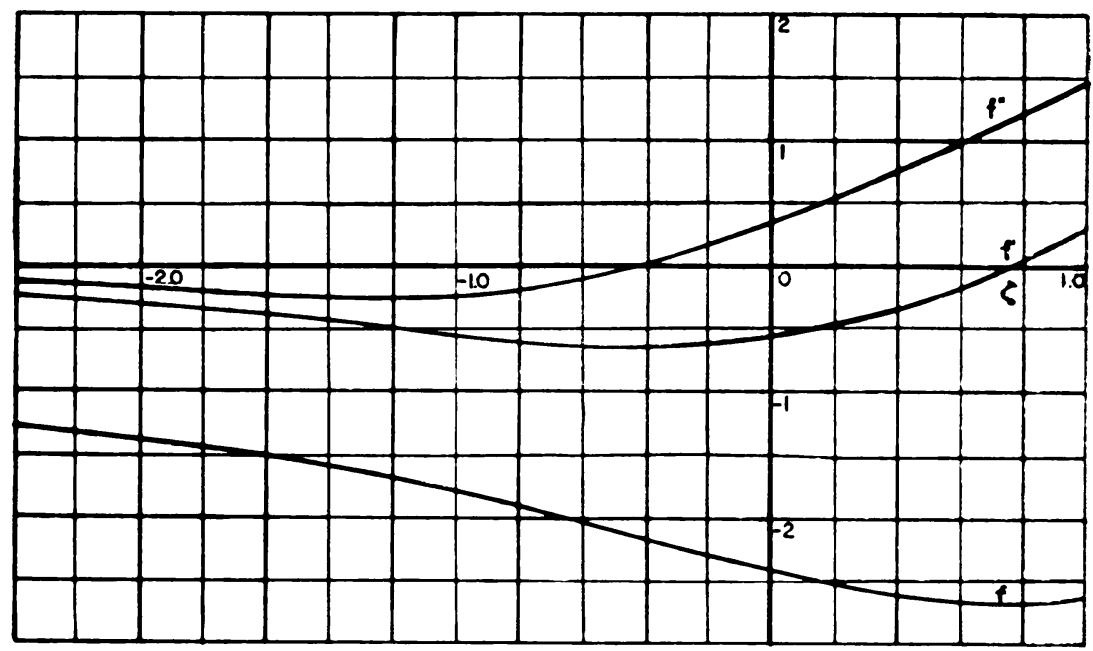

FIG. 3. The functions $f, f^{\prime}$ and $f^{\prime \prime}$

The main symptoms of a wrong choice of $n$ will appear in the continuation from point $B^{\prime}$. Depending upon the choice of $n$ one will have curves either above or below the desired curve $B^{\prime} C$. The integral curves which lie above $B^{\prime} C$ will intersect the dotted line of Fig. 1; they will therefore represent flows with a limiting line. Those integral curves which lie below $B^{\prime} C$ will cross the sonic line $B^{\prime} A$ and enter point $A$ with supersonic velocities. One will expect that in this case one does not enter point $A$ along that integral curve representing zero downwash velocities along the $x$-axis.

Are such flow patterns physically possible? Let us consider the first case in which a limiting line occurs. In this case, the limiting line is formed by an envelope of expansion waves. Such a configuration of Mach waves in which one expansion wave starts from a neighboring one in the interior of the flow may be considered as arising from a series of thin knife edges placed along the limiting line (see Ref. 3). A flow pattern of this kind arises for a value of $n$ for which the perturbation velocities vanish rather rapidly as one goes to infinity.

The integral curve which lies below the desired one, on the other hand, represents a 
flow in which the perturbation velocities decrease very slowly towards infinity. Here the flow will correspond to that over a semi-infinite body whose contour is shaped in such a way that the expansion waves originating from the body are never sufficient to produce supersonic velocities up to a very great distance from the body. In this case the limiting Mach wave does not lie in the finite portion of the physical plane, so that all Mach waves from the body will reach the sonic line.

According to this description the flow patterns of the first type have a high density of rarefaction waves which can be produced only by a limiting line, while the flow patterns of the second have a sparse distribution which requires a carefully shaped body which extends to infinity. The desired flow represents the transition between the above two types of flows of opposite character.

As mentioned previously it was found more practical to carry out the numerical computation in terms of the differential equation for $f$. The most satisfactory procedure for the determination of $n$ was to begin the integration from both points $A$ and $C$ and then match the two resulting integrations at a point intermediate to the two points using the $s, t$-representation. The solutions near the singular points are found by a suitable series representation. Within the accuracy of the numerical computation the value of $\mathbf{n}$ was found to be $4 / 7$. The resulting function $f$ and its derivatives $f^{\prime}$ and $f^{\prime \prime}$ are shown in Fig. 3. The streamline pattern for the flow represented by the basic singularity is shown in Fig. 4.

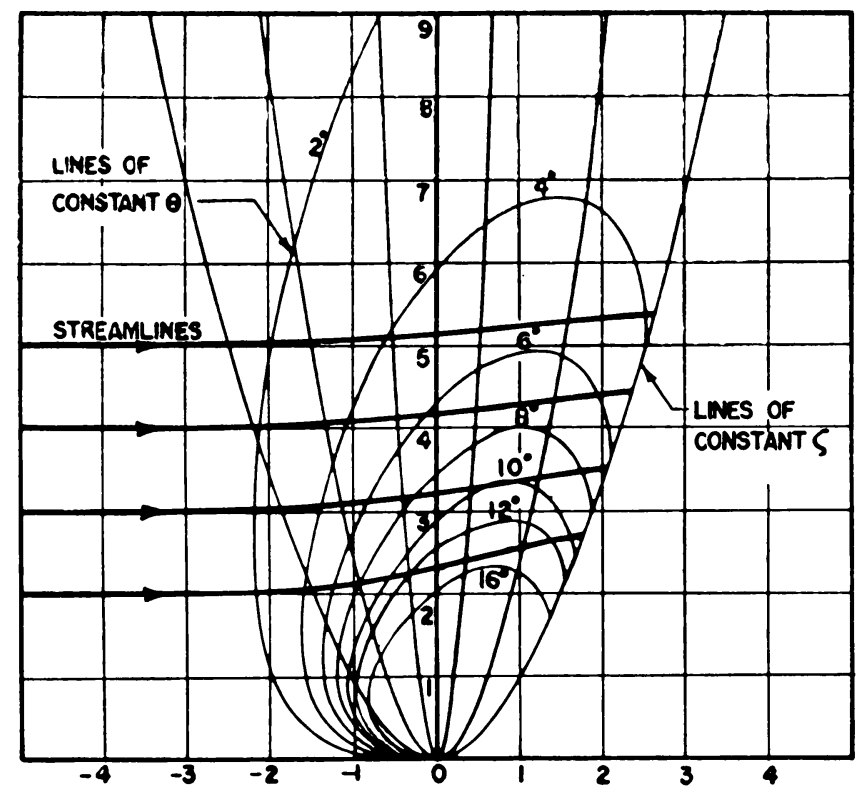

Fig. 4. Lines of constant $\theta$ and the streamlines for the flow represented by the basic singularity.

6. The final results. The basic singularity given by Eq. 3 with $n=4 / 7$ may now be used to study the perturbation velocities at infinity caused by an axial-symmetric body with a free stream Mach number one. (Here the role of the basic singularity is analogous to that of a doublet singularity in the case of a body in an incompressible flow.) 
The results show that as one proceeds farther and farther from the axis of symmetry along lines of constant $\zeta$, where $\zeta=(\kappa+1)^{-1 / 3} x y^{-4 / 7}$ the perturbation velocities $\varphi_{x}$ and $\varphi_{y}$ decrease as $y^{-6 / 7}$ and $y^{-9 / 7}$ respectively. (In the two-dimensional case these velocities decreased as $y^{-2 / 5}$ and $y^{-3 / 5}$ as one traveled outward along the curve $x y^{-4 / 5}=$ constant. Cf. Ref. 1.)

In Fig. 5 is shown the distribution of the pressure coefficient $C_{p}$ and the streamline

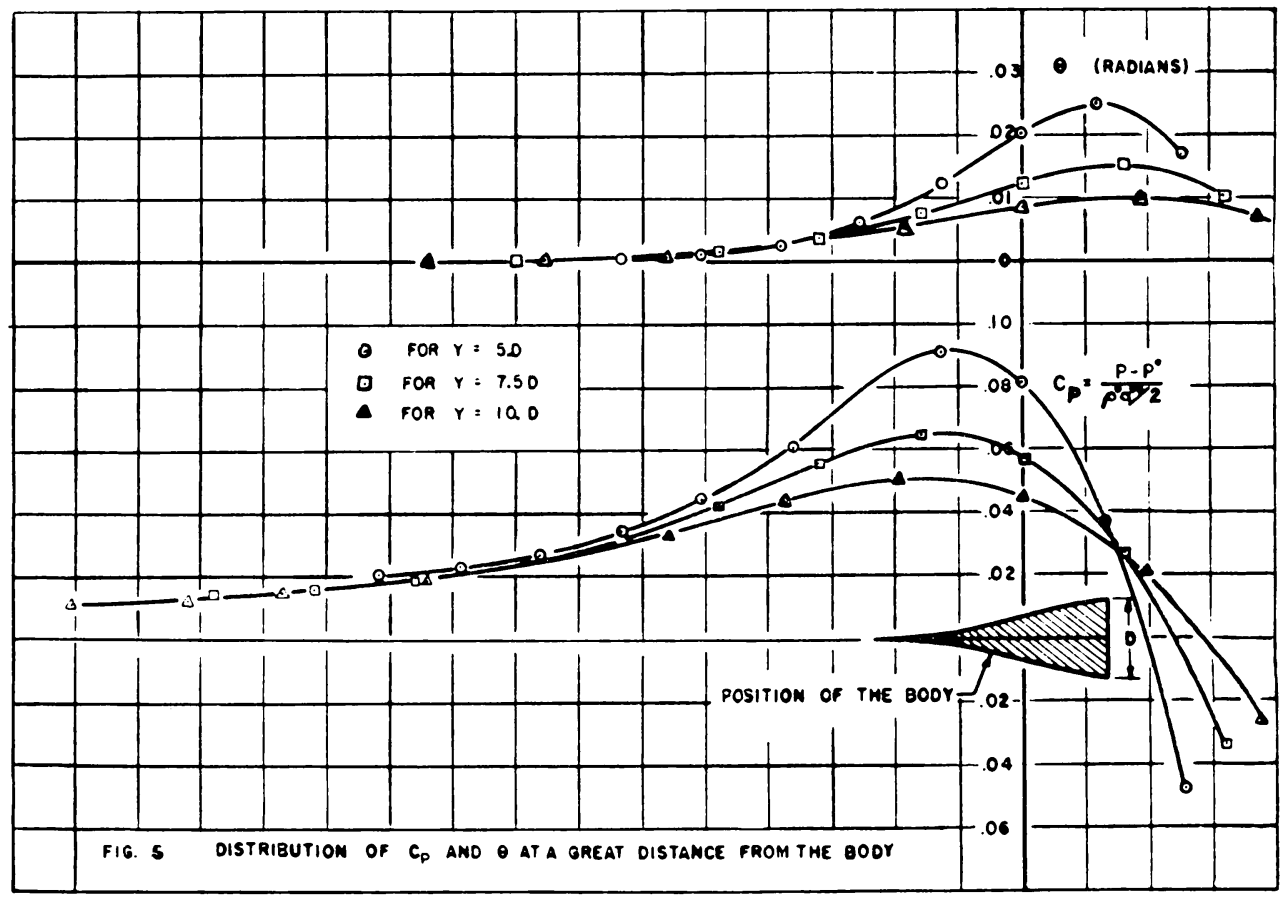

deflection $\theta$ along a horizontal line at different distances from the axis. Here $C_{p}=$ $2\left(p-p^{*}\right) / \rho^{*} a^{* 2}$, where $p$ and $\rho$ are the local pressure and density and the asterisk denotes the sonic free stream conditions.

It is of further interest to determine a body shape which produces a flow at infinity given by the basic singularity. An approximate construction of such a body has been carried out in Ref. 4, and the resulting contour is shown in Fig. 5.

\section{REFERENCES}

1. G. Guderley, Singularities at the sonic velocity, Wright Field Report F-TR-1171-ND, June 1948.

2. G. Guderley and H. Yoshihara: The flow over a wedge profile at Mach number 1, USAF Technical Report No. 5783, July 1949.

3. G. Guderley, Rueckkehrkanten in ebener kompressibler Potential Stroemung, ZAMM 22, 121-126 (1942).

4. G. Guderley and H. Yoshihara, On axial-symmetric transonic flow patterns, USAF Technical Report No. 5797, September 1949. 